Acta Poetica 25-1

PRIMAVERA

2004

\title{
La inefable naturaleza de la mente. Versiones e interpretaciones
}

\section{Bertha Aceves}

Las versiones que se han escrito y se seguirán escribiendo en torno a seis versos, provenientes de una tradición reservada a un grupo de iniciados, manifiestan las limitaciones del lenguaje para transmitir el mensaje contenido en estas seis líneas. Cada lector, de acuerdo con su entorno histórico y social, produce un nuevo texto, que se acerca o se aleja del sentido primario del manuscrito.

The different versions that have been made - and will continue to be made - of six verses that are part of a tradition reserved to a group of initiates, clearly show the limitations of language's hability to convey the message contained within these six lines. Each reader, according to his or her own social and historical context, will produce a new text that may come closer to or may drift away from the primary sense of the manuscript. 


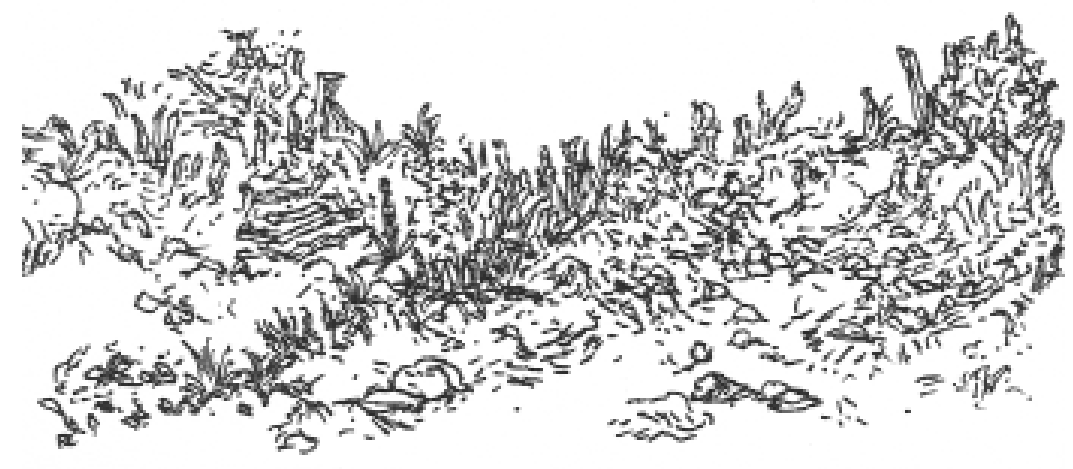


Acta Poetica 25-1

PRIMAVERA

2004

Bertha Aceves

\section{La inefable naturaleza de la mente. Versiones e interpretaciones}

All things have the nature of mind. Mind is the chief and takes the lead. If the mind is clear, whatever you do or say will bring happiness that will follow you like shadow

DHAMMAPADA

Las múltiples interpretaciones que se han propuesto para la lectura de un texto budista de seis versos llamado La Canción del Cuco de la Total Presencia o Los Seis Versos Vajra, expresan la dificultad de comprender los significados contenidos en un lenguaje altamente cifrado con el cual fue escrito. Estos versos fueron escritos en el siglo viII en tibetano, aunque provienen de una tradición oral anterior, y estaban dirigidos a un público muy limitado de iniciados en la práctica del Dzogchén, enseñanza budista que se explica más adelante.

El título del texto es diverso, dado que "es muy común que los textos del Dzogchén tengan diversos títulos para un solo trabajo" (Karmay, 1988, 42). De tal manera que, cuando el título es Rig-pa'i khu-byug, se traduce, según Keith Dowman como La Canción del Cuco de la Total Presencia (Dowman, b). El cuco, en la tradición tibetana, es un pájaro azul, el heral- 
do del verano, que proviene del sur de la India y arriba al Tíbet como el mensajero del término del invierno; tal como acontece con el arribo del cuco, cuando las plantas y los árboles crecen y se nutren los animales, de la misma manera, al escuchar las enseñanzas del Dzogchén, se despierta la propia mente y entra en su presencia espontáneamente surgida, no dual, sin límites, clara y absoluta, como el azul infinito del espacio.

Cuando se titula como rDo-je tshig drug, Los Seis Versos del Vajra, el vajra en sánscrito o dorje en tibetano es un símbolo con múltiples significados, pero el más común es el de diamante; así, estos versos poseen el indestructible poder del diamante, y por ello cortan con todos los obstáculos que impiden a la mente reconocer su prístina naturaleza no dual. Son tan valiosos y límpidos como esta piedra preciosa.

\section{Manuscritos de La Canción del Cuco de la Total Presencia}

La importancia en el pensamiento budista de este texto es evidente, pues es uno de los textos raíz para las enseñanzas del Dzogchén. El texto tiene tres fuentes, la primera son unos documentos hallados en las cuevas de Tun-huang o Dunhuang en China. ${ }^{1}$ Estas cuevas están situadas al oeste de Xian, la antigua capital china de la Dinastía Tang. La importancia de este sitio radica en que se encontraba en el cruce de dos caminos, en la Ruta de la Seda, y en su cercanía a la antigua capital. En esta área se encuentran 492 cuevas, que guardan aún estatuas y frescos; los manuscritos, textiles y otros objetos fueron ex-

\footnotetext{
${ }^{1}$ Este texto se publica y traduce en el libro The Great Perfection (Karmay, 1988). Los textos encontrados en Tun-huang son de tres tipos: administrativos, budistas y pre budistas (textos rituales del Bön). El material budista proporciona una muy buena idea de la naturaleza del budismo tibetano en ese tiempo, esto es, desde mediados del siglo ocho hasta el siglo diez, cuando la cueva biblioteca fue sellada. Uno de estos textos es el de los seis versos de La Canción del Cuco de la Total Presencia.
} 
traídos de éstas. La Cueva 17 llamada "La Cueva Biblioteca", que había sido clausurada en el siglo x, fue descubierta accidentalmente por un monje taoísta, Wang Yuanlu, el 22 de junio de 1900. En ella se encontraron más de 50,000 sutras, documentos históricos, textiles y pinturas que pertenecieron al periodo contenido entre los siglos IV al x. Fue un sorprendente descubrimiento que ha permitido tener información de fuentes originales budistas.

El texto de los seis versos está catalogado como Documento I (IOL 647) y "contiene cinco folios en el común formato tibetano. (...) El estilo del manuscrito es la escritura 'bru ma. Es el estilo temprano de la escritura que más tarde se hizo popular en ciertas regiones de Kham y Amdo" (Karmay, 42). Según Karmay los títulos dados a los seis versos son tres y los traduce como El Cuco del Intelecto, El ornamento de la apariencia que trae la autorrealización y Los Seis Versos del Vajra $^{2}$ (Karmay, 42).

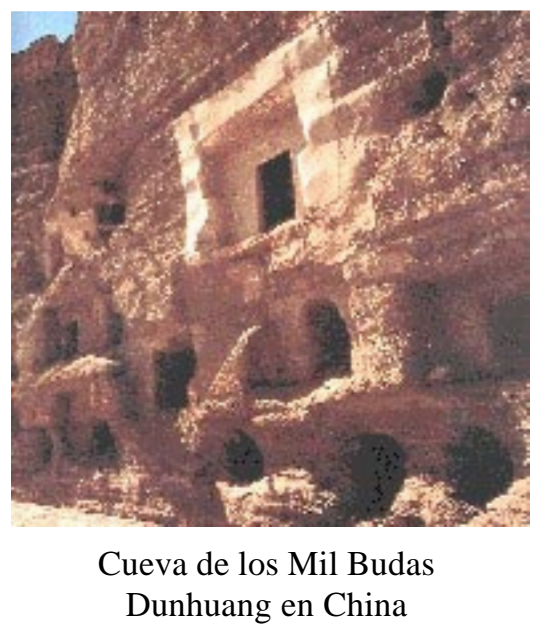

\footnotetext{
${ }^{2}$ En inglés: "Cuckoo of Intellect", "Ornament of the Appearance which brings one the realization" y "Six vajra words". En tibetano: rig pa'i khu byug dpe/ rig byed snang ba'i rgyan ni don/ rdo rje tshig drug ni grangs.
} 
La segunda fuente es un documento llamado Kun byed rgyal po. ${ }^{3}$ De hecho, los seis versos recogidos en los documentos encontrados en Tun-huang, según lo señala Karmay, "constituyen el capítulo treinta y uno del documento Kun byed rgyal po, con el título de rDo rje Tshig Drug (Los Seis Versos Vajra)" (Karmay, 47). El texto compilado en el Kun byed rgyal po, según la traducción de Namkhai Norbu, es El Cuco del Estado de la Presencia. Un Signo de Buena Fortuna y Gloria (bKra shis pa'i dial rig pa'i khu byug) (Chögyal Namkhai b, 1996, 78). Pese a que el título tiene algunas variaciones de la fuente primera a la segunda, el cuerpo del texto, los seis versos, es idéntico.

El Kun byed rgyal po, a su vez, es parte de otro documento tibetano, titulado Vairo Drabag (Bai ro 'dra 'bag), que es un manuscrito atribuido al gran maestro y traductor tibetano Bagor Vairochana. Dice la leyenda que, cuando Vairochana estaba a punto de morir, sus discípulos le pidieron que narrara su historia y su viaje a la India, así como los estudios sobre el budismo, en especial el del Atiyoga (el Dzogchén pertenece a esta disciplina). "El 'Dra'bag se presenta como la respuesta oral a los requerimientos de sus siete discípulos, quienes lo escribieron".

La tercera fuente corresponde al rNying-ma'i rgyud'bum. En este manuscrito se recogen las escrituras del canon Niyngmapa (Norbu, 1996, 139) ( Myrdhing, 216).

Según la segunda y la tercera fuente, el texto de La Canción... pertenece al traductor tibetano Bagor Vairochana. ${ }^{4}$

\footnotetext{
${ }^{3}$ A partir de este texto, Chögyal Namkhai Norbu ha traducido y publicado el documento de los seis versos en distintas obras suyas, como se explica adelante.

${ }^{4}$ Bagor Vairochana uno de los siete tibetanos a quienes Shantaraksita ordenó como primeros monjes tibetanos, en el primer monasterio del Tíbet, Samye, fundado en año 775 por el Rey Trisong Detsen. Este rey fue quien introdujo por primera vez el budismo al Tíbet, y trae de la India al maestro Shantaraksita y al yogui Patmasambhava para difundir las enseñanzas del budismo, hasta entonces desconocidas en ese país.
} 


\section{El Dzogchén "La Gran Perfección"}

El término Dzogchén quiere decir "la total perfección" y "la total plenitud". Dice Adriano Clemente que: "a pesar de que en el curso de la historia del Tíbet, el Dzogchén fue introducido y expandido por el país por las dos grandes tradiciones del Budismo y Bön, el Dzogchén en sí mismo no puede ser calificado como una religión o una tradición filosófica. Antes bien, es un camino completo del conocimiento del estado individual del ser, más allá de los límites de cualquier creencia religiosa o cultura. Sin haberse convertido en una secta, el Dzogchén ha permanecido como una enseñanza directa porque, con el paso de los años, ha mantenido la pureza y autenticidad de la transmisión, incompatible con las instrucciones religiosas mas formales y estructuradas" (Norbu 1996, 13).

Sin embargo, cabe decir que, en la práctica del budismo tibetano, ${ }^{5}$ el Dzogchén fue una de las enseñanzas más secretas y estuvo reservada para quienes alcanzaron los más altos grados en las enseñanzas del tantra, que son los tantras internos del Mahayoga, Anuyoga y Atiyoga. ${ }^{6}$

\section{Origen de La Canción del Cuco}

La Canción del Cuco de la Total Presencia pertenece a la tradición de la escuela Nyingma, "los antiguos"; esta escuela es la primera que se organiza en el Tíbet, a partir de la fundación del monasterio de Samye. Según las fuentes antes indicadas,

\footnotetext{
${ }^{5}$ El budismo que fuera introducido por el rey Trisong Detsen (742-797 D.C.), después decrece y casi desaparece; sin embargo, nuevamente se reestablece y predomina como la religión aceptada desde el siglo XII hasta la invasión china en 1959. Las escuelas del budismo que consecutivamente se establecieron fueron la Nyingmapa, la Kagyupa, la Sakyapa y la Gelupa.

6 "El Mahayoga aparece como el milagro de la conciencia alerta. Anuyoga aparece como la expresión del poder de la conciencia intrínseca. Atiyoga es la conciencia alerta, que se manifiesta por y en sí misma". Dudjom Rinpoche, The Nyingma School, vol. II, 358.
} 


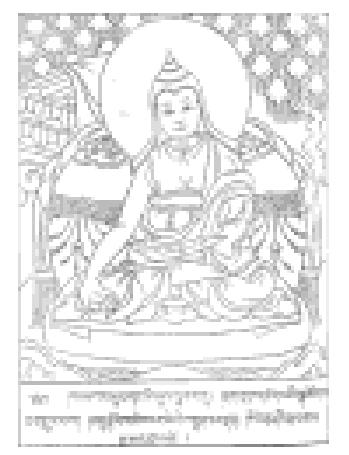

Bagor Vairochana

este texto fue recogido por un monje tibetano llamado Bagor Vairochana, quien fue enviado a Oddiyana, lugar mítico situado en el centro de la India, por el rey Trisong Detsen para obtener los textos de las enseñanzas Dzogchén y traducirlos al tibetano.

En Oddiyana, Vairochana conoció al maestro Shri Singha, quien le enseñó tanto los sutras como las enseñanzas Dzogchén, estas últimas durante la noche, a causa de la prohibición impuesta por el rey de Oddiyana de transmitir estas prácticas a los tibetanos, por ser secretas. De regreso al Tíbet escribe y traduce al tibetano las enseñanzas Dzogchén, y las trasmite al rey y a unos cuantos otros elegidos. El texto de Los Seis Versos Vajra fue de hecho una de las primeras prácticas impartidas en el Tíbet.

La transmisión del Dzogchén, que recibió Bagor Vairochana de su maestro Shri Singha, consistía en enseñanzas que habían sido comunicadas oralmente a través de varias generaciones anteriores, sin interrupción, desde Garab Dorje. Este maestro, según la tradición nyingma, había nacido trescientos sesenta y seis años después de la muerte del Buda (483 a. C.), esto es, en el año de 117 a. C, y manifestó desde niño grandes y sorprendentes cualidades. 


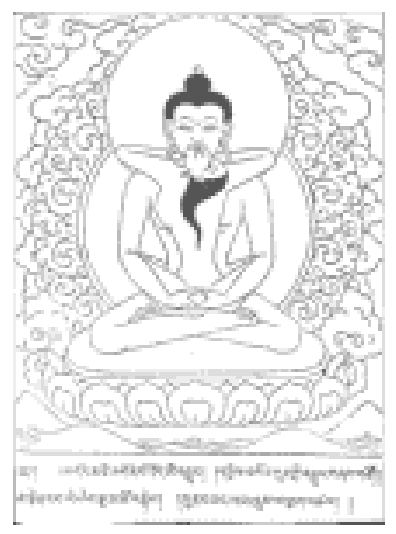

Samantabhadra y Samantabhadri.

Representan el estado de la no dualidad

En el Dzogchén se establecen diferentes linajes, pero todos ellos empiezan con Garab Dorje, y llegan a Shri Singha, quien fuera el maestro de Bagor Vairochana. Los linajes en el budismo son primordiales porque manifiestan la pureza y autenticidad de las enseñanzas, dado que éstas se impartieron oralmente, por mucho tiempo, y aun después de fijar los textos en escritura.

A su vez Garab Dorje, según el árbol del linaje nyingma, desciende del buda primordial Samantabhadra, en sánscrito, (Kuntu Zangpo, en tibetano) es la representación de la "naturaleza no dual" de "la mente primordial"; por ello se le personifica desnudo. "Representa La Conciencia del Conocimiento que es el universo, su nombre significa 'El todo Bueno', porque su bondad es trascendental y por encima de lo moral. Representa en sí mismo el Dzogchén en su fase de resultado" (Dowman 1994, 143). Después le siguen Garab Dorje y los siguientes maestros, hasta llegar a Vairochana.

Namkhai Norbu, al definir etimológicamente el título de Kunjed Gyalpo, documento en el cual se encuentran los seis versos, precisa más ampliamente el simbolismo de Samanta- 
bhadra: "Literalmente (Kunjed Gyalpo) es la traducción de 'el rey de todo lo creado', en realidad Kunjed Gyalpo es sinónimo de Samantabhadra (...). En un nivel más profundo, el verdadero significado del término kun es 'todo', que es el vacío, si se considera que la última naturaleza de todos los fenómenos es la condición original de la pureza ( $k a$ dag) o el vacío. Jed es un verbo que significa 'hacer' o 'actuar', se refiere a la energía natural del vacío que, al ser dotada con movimiento y actividad, se manifiesta como claridad ( $g s a l b a$ ), visión (snag $b a$ ) y una pura e instantánea presencia (rig pa) (...). El término gyalpo o 'rey', significa el principio del conocimiento que corresponde a la total perfección del estado primordial del estado del Ati Dzogpa Chenpo: nuestra auténtica y original condición" (Norbu y Clemente 1999, 14).

Así, los principales estudios coinciden en que la trasmisión oral de los versos fue concedida a Vairochana por Shri Singha en Oddiyana, y que éste la escribe en tibetano en el siglo octavo. Pero su antigüedad oral no se puede precisar con exactitud, porque la existencia de Garab Dorje se pierde entre la historia y la leyenda de este maestro. ${ }^{7}$

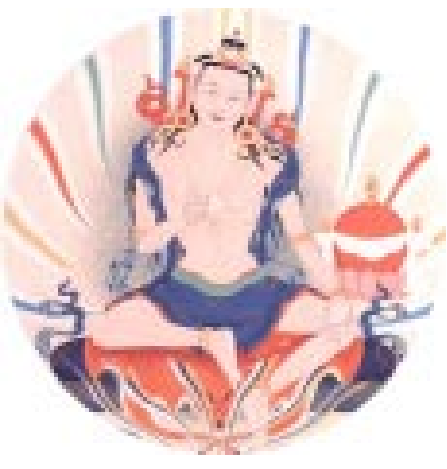

Garab Dorje

\footnotetext{
${ }^{7}$ Ver: Norbu y Clemente 1999, 26-30; Myrdhing, 205-213; Dowman, www. keithdowman.net. Dudjom, "The lineage of Atiyoga, The Great Perfection", 1991, 491-527.
} 
El texto, en caligrafía tibetana, escrito por Chöyal Namkhai Norbu, es así:

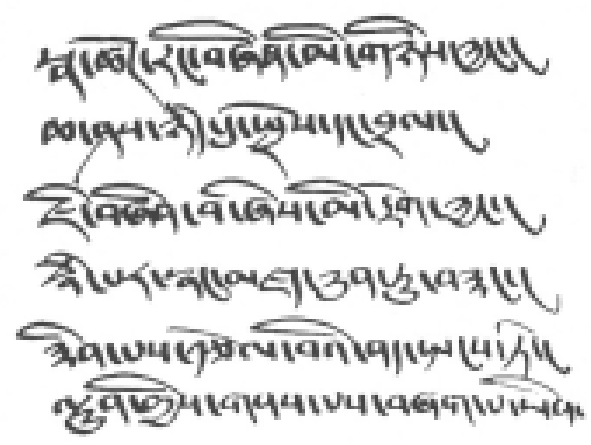

Transliterado del tibetano se lee así:

1 sNa tshogs rang bzhin mi gnyis kyang

2 cha shas nyid du spros dang bral.

3 ji bzhin pa zhes mi rtog kyang

4 rNam bar snang mdzad kun tu bzang

5 zin bas rtsol ba'i nad spangs te

6 lhun gyis gnas pas bzhag pa yin.

La primera interpretación libre hecha por Brian Beresford y John Shane se publica en un libro de Chögyal Namkhai Norbu, The Crystal and the Way of Light. Sutra, Tantra and Dzogchen, en 1986:

The Six Vajra Verses

Although apparent phenomena manifest as diversity yet this diversity is non-dual, and of all the multiplicity 
of individual things that exist

none can be confined in a limited concept.

Staying free from the trap of any attempt to say it's 'like this', or 'like that', it becomes clear that all manifested forms are aspects of the infinite formless, and, indivisible form it, are self-perfected.

Seeing that everything is self-perfected from the very beginning,

the disease of striving for any achievement Comes to an end of its own accord, and just remaining in the natural state as it is, the presence of non-dual contemplation continuously, spontaneously arises.

La versión al español que presento pertenece a la traducción del libro anterior hecha por Elías Capriles en 1996: El cristal y la vía de la luz. Sutra, Tantra y Dzogchén:

Los Seis Versos Vajra

Aunque fenómenos aparentes se manifiestan como diversidad, esta diversidad es no dual, y de toda la multiplicidad existente de cosas individuales ninguna puede ser confinada en un concepto limitado.

Cuando nos encontramos libres de la trampa constituida por los intentos de decir "es asî" o "es asá", se hace claro que todas las formas manifiestas son aspectos del infinito sin forma e, indivisibles de él, se encuentran [ya] autoperfeccionadas. 
Cuando vemos que cada cosa está [ ya] autoperfeccionada, la enfermedad de esforzarse por un logro, rendida

se desploma [por sí misma],

$\mathrm{y}$, permaneciendo simplemente en el Estado

natural tal como es,

la Presencia (de la) Contemplación no dual

surge espontáneamente en forma continua.

En una obra posterior de Namkhai Norbu (Dzogchen. The Self-Perfected State, 1996; editada en italiano en 1986 y traducida al inglés por John Shane) se publica una traducción más apegada al texto tibetano; sin embargo, la explicación expande los seis versos en once versos:

\section{The Six Vajra Verses}

The nature of phenomena is non-dual, but each one, in its own state, is beyond the limits of the mind.

There is no concept that can define the condition of "what is"

but vision nevertheless manifests: all is good.

Everything has already been accomplished, and so, having overcome the sickness of effort, one finds oneself in the self-perfected: this is contemplation.

La última traducción fue hecha del italiano (de una edición de 1997) al inglés en 1999. En esta versión los seis versos originales se conservan, al igual que la traducción que presenta Keith Dowman, sin embargo, ambas versiones emplean un vocabulario diferente:

The nature of the variety of phenomena is non-dual Yet each phenomenon is beyond the limits of the mind The authentic condition "as it is" does not become a concept 
Yet it manifests totally in form, always good

All being already perfect, overcome the sickness of effort.

And remain naturally in self-perfection: this is contemplation.

La traducción que presenta el profesor Samthen Gyaltsen Karmay, en The Great Perfection, es la siguiente:

All the varieties of phenomenal existence as a whole do not in reality differ one from another.

Individually also they are beyond conceptualization.

Although as «suchness» there is no mental discursiveness (with regard to them)

Kun-tu bzang po shines forth in all forms.

Abandon all the malady of striving, for one has already acquired it all.

One leaves it as it is with spontaneity.

El maestro, yogui, escritor y traductor del tibetano al inglés, Keith Dowman, presenta la siguiente versión:

The nature of multiplicity is nondual and things in themselves are pure and simple; being here and now is thought-free and it shines out in all forms, always all good; it is already perfect, so the striving sickness is avoided and spontaneity is constantly present.

John Myrdhing Reynolds (Vajranath) presenta la siguiente versión, con base en los Seis Versos Vajra traducidos por Namkhai Norbu.

(1) Even though (kyang) the nature of the diversity (of all phenomena) (sna-tshogs rang-bzhin) is without any duality (mi gnyis),

(2) In the terms of the individuality of the things themselves (chashas nyid $\mathrm{du}$ ), they are free of any conceptual elaborations 
(made by mind)

(spros dang bral)

(3) Even though (kyang) there exists no thought or conception ( $m i$ rtog) of what is called (zhes) the state of being just as it is (jibzhin-pa),

(4) These various appearances which are created (rnam-par snang $m d z a d)$

are but manifestations of Samantabhadra (the Primordial State)

(kun tu bzang).

(5) Since everything is complete in itself (zin-pas), one comes to abandon the illness (or obsession with) of efforts (rtsol-ba'i nad spangs te),

(6) And thus one continues (bzhag-pa yin) spontaneously (lhun gyis) in the calm state of contemplation ( gnas-pa).

Significado, Palabras: La Presencia Absoluta

Como se ha dicho anteriormente, las enseñanzas budistas son transmitidas oral y directamente del maestro al discípulo. Primero se recibía la transmisión o sea la autorización, el conferir al otro el poder para estudiar un texto o hacer un tipo de práctica. Se enseñaba el texto raíz y después se explicaban los significados que éste contenía, y si era el caso, el tipo de práctica meditativa que se debería de realizar. De hecho el manuscrito de Tun-huang contiene dos trabajos: el texto básico (rtsa ba), y el comentario (' grel ba).

Los textos escritos no podían ser leídos por cualquier practicante, como en occidente, sino que se debería tener la aprobación del maestro, quien le confería primero la transferencia del linaje de la práctica, sin ninguna variación, tal cual él mismo la recibió, para conservar su pureza. Esto se puede constatar en las tres fuentes distintas en las cuales se encontró el documento de Los Seis Versos del Vajra, el texto no tuvo ninguna variante. Por ello, el texto escrito contenía no sólo significados 
lingüísticos, sino la energía de todo el linaje; en sí mismo, el texto tenía un poder más allá del lenguaje.

Las diferencias provienen de las interpretaciones, que pretenden explicar con mayor claridad los contenidos del texto. Como se observa en cada una de las interpretaciones anteriores, la diferencia es grande; cada una de ellas escribe su propio texto. En esto intervienen varios factores: por un lado, está el lenguaje altamente cifrado que no permite una traducción única; también, hay que considerar que quienes recibían la transmisión de una práctica o texto, eran budistas y que habían obtenido el más alto grado de esta filosofía y el mayor grado de estabilidad meditativa. Esto es, un público especializado que conocía ampliamente el contexto cultural del que provenía la transmisión y en el cual se impartían estas enseñanzas. Por ello, las connotaciones, las referencias y el simbolismo implícito en el texto son muy extensos, y el sentido que tiene cada verso, cada término, está vinculado con los múltiples códigos presentes en el pensamiento budista.

Tomo por ejemplo el título de Rig-pa' khu-byu. Khun-byu, el cuco, nombre que aparentemente no presenta problema de interpretación, pero que, como se ha explicado anteriormente es un símbolo rico en significados. Rig-pa es traducido por Keith Dowman como The Cuckoo's Song of Total Presence; por Namkhai Norbu (1966) The Cukoo of The State of Presence, y Norbu (1999) The Cukoo of the Presence; por Samthen Gyaltsen Karmay, The Cukoo of Intelect; y por John Myrdhing Reynolds, The Cukoo of Awareness.

La traducción de Rig-pa como "la presencia total", "el estado de la presencia", "el intelecto" y "la conciencia" es extremadamente limitada. Rig-pa, en el contexto del Dzogchén, es un término utilizado para denotar una experiencia meditativa a la cual accede el practicante. Se refiere al conocimiento directo e intuitivo de la condición primordial, que permanece como una presencia viva: "un estado de presencia, claridad y espa- 
ciosidad ilimitada" (Norbu 1996, 136). Ésta puede describirse en distintas formas: como un estado de contemplación, luminosidad, espaciosidad, incesante vacío, etc. Pero estos son sólo términos que circundan el núcleo central de una experiencia: la presencia constante y absoluta de la naturaleza más intrínseca, primordial y fundamental de la mente, que es Rig-pa. Así, hay niveles de la comprensión, entendimiento o intuición mental que no pueden ser formulados verbalmente. Las palabras tienen un límite, según dice el último precepto del Dzogchén: "Éste se sustenta en el significado y no en las palabras", por ello, lo que permanece es el significado, las interpretaciones prosiguen.

\section{Referencias}

CAPRILES, Elías, Budismo y Dzogchén. La doctrina del Buda y el Vehículo Supremo del budismo tibetano, Barcelona, La Llave, 2000.

Dharmmapada [trad. del Tibetan por Pâli por dGe'-dun Chos'phel], trad. por Dharma Publishing Staff., Berkeley, California, Dharma Publishing, 1985.

Dowman, Keith (ed. y trad.), The Flight of the Garuda, Boston, Wisdom Publications, 1994.

-b) www.keithdowman.net

Dudjom, Jikdre Yeshe Dorje, The Nyingma School of Tibetan

Buddhism. Its Fundamentals and History, 2 vols., trad. y ed. por Gyume Dorje y Matthew Kapstein, Boston, Wisdom Publications, 1991.

Karmay, Samthen G., The Great Perfection (rDzogs Chen). A Philosophical and Meditative Teaching in Tibetan Buddhism, Leiden, Brill, 1988. 
Myrdhing Reynolds, John (ed. y trad.), The Golden Letters, pról. de Namkhai Norbu, Ithaca, NY, Rinpoche Snow Lion Publications, 1996.

Norbu, Chöyal Namkhai, The Crystal and the Way of Light. Sutra, Tantra and Dzogchen [Penguin Books, Ltd, 1986], ed. por John Shane; $2^{\text {nd }}$ d, Itaca, NY, Snow Lion Publications, 2000.

- El cristal y la vía de la luz. Sutra, Tantra y Dzogchén, trad. de Elías Capriles, Barcelona, Kairós, 1996.

- b) Dzogchen. The Self-Perfected State [1986], ed., Adriano Clemente, trad. John Shane, Itaca, NY, Snow Lion Publications, 1996.

- y Adriano Clemente, The Supreme Source. The Fundamental Tantra of the Dzogchen Semde. Kunjed Gyalpo [1997], trad. por Andrew Lukianowicz, Ithaca, NY, Snow Lion Publications, 1999. 